\title{
Possible Role of Advanced Glycation End Products (AGEs) In the Clinical Course of Prostate Neoplasies Among Patients with and without Type 2 Diabetes Mellitus
}

\author{
Gamze Akkus ${ }^{1}$, Volkan Izol ${ }^{1}$, Fesih OK ${ }^{1}$, Mehtap Evran ${ }^{1}$, Merve Inceman ${ }^{1}$, Seyda \\ Erdogan $^{1}$, Halil Mahir Kaplan ${ }^{1}$, Murat Sert ${ }^{1}$, and Tamer Tetiker ${ }^{1}$ \\ ${ }^{1}$ Cukurova University
}

August 23, 2020

\begin{abstract}
Aim: The cognate receptor expression of AGE (RAGE; receptor for AGE) on malignant tissues in diabetic patients has been suggested as a co-factor in determining the clinical course and prognosis. We aimed to search this relationship between RAGE expression and clinicopathological features of prostate neoplasia. Methods: A total of 197 patients; 64 (diabetic n=24; nondiabetic $\mathrm{n}=40$ ) with benign prostate hyperplasia, and 133 (diabetic $\mathrm{n}=71$; non-diabetic $\mathrm{n}=62$ ) with localized (LPCa) $/$ metastatic prostate cancer (MetPCa) were included the study. The expression of RAGE was studied by immunohistochemically on prostate specimens. The RAGE score was assessed in the specimens according to the extent of immunoreactivity and staining intensity. Results: The RAGE scores of BPH patients (diabetic and non-diabetic) were found as negative. Patients with both LPCa and MetPCa showed significantly higher scores, respectively (LPCa and MetPCa vs. BPH; p<0.01). RAGE scores of diabetic patients with LPCa and MetPCa were found to be $4.71 \pm 3.14$ and $4.97 \pm 3.69$, respectively. RAGE scores of the non-diabetic patients who had LPCa and MetPCa were 1.52 \pm 1.87 and 1.69 \pm 1.58 , respectively. When compared both groups with LPCa, RAGE scores of the diabetic patients were significantly higher than that of the non-diabetics $(\mathrm{p}=0.01)$. Similar results were revealed as for the patients with MetPCa (4.97 \pm 3.69 vs. $1.69 \pm 1.58$ (diabetic vs. non-diabetic), respectively ( $\mathrm{p}<0.01)$. Conclusion: We found a high rate of RAGE expression in malign prostate neoplasias to the BPH. Furthermore, as expected, higher scores were demonstrated in those with diabetes than non-diabetics. Disease progression and survival parameters were worse in the patients with high RAGE levels. RAGE may be useful in the diagnosis of prostate cancer and in determining its prognosis.
\end{abstract}

\section{Possible Role of Advanced Glycation End Products (AGEs) In the Clinical Course of Prostate Neoplasies Among Patients with and without Type 2 Diabetes Mellitus}

Aim: The cognate receptor expression of AGE (RAGE; receptor for AGE) on malignant tissues in diabetic patients has been suggested as a co-factor in determining the clinical course and prognosis. We aimed to search this relationship between RAGE expression and clinicopathological features of prostate neoplasia.

Methods: Atotal of 197 patients; 64 (diabetic $\mathrm{n}=24$; non-diabetic $\mathrm{n}=40$ ) with benign prostate hyperplasia, and 133 (diabetic $\mathrm{n}=71$; non-diabetic $\mathrm{n}=62$ ) with localized ( $\mathrm{LPCa}$ )/metastatic prostate cancer (MetPCa) were included the study. The expression of RAGE was studied by immunohistochemically on prostate specimens. The RAGE score was assessed in the specimens according to the extent of immunoreactivity and staining intensity.

Results: The RAGE scores of BPH patients (diabetic and non-diabetic) were found as negative. Patients with both LPCa and MetPCa showed significantly higher scores, respectively (LPCa and MetPCa vs. BPH; $\mathrm{p}<0.01$ ). RAGE scores of diabetic patients with LPCa and MetPCa were found to be $4.71 \pm 3.14$ and $4.97 \pm 3.69$, respectively. RAGE scores of the non-diabetic patients who had LPCa and MetPCa were 
$1.52 \pm 1.87$ and $1.69 \pm 1.58$, respectively. When compared both groups with LPCa, RAGE scores of the diabetic patients were significantly higher than that of the non-diabetics $(\mathrm{p}=0.01)$. Similar results were revealed as for the patients with MetPCa (4.97 \pm 3.69 vs. $1.69 \pm 1.58$ (diabetic vs. non-diabetic), respectively ( $<<0.01)$.

Conclusion: We found a high rate of RAGE expression in malign prostate neoplasias to the BPH. Furthermore, as expected, higher scores were demonstrated in those with diabetes than non-diabetics. Disease progression and survival parameters were worse in the patients with high RAGE levels. RAGE may be useful in the diagnosis of prostate cancer and in determining its prognosis.

Keywords : AGE, Type 2 Diabetes Mellitus, Prostate, Carcinogenesis

\section{What's known}

- There is a complex relationship between type 2 diabetes and prostate cancer development and prognosis.

- Advanced glycation end products have been shown to be associated with prostate cancer development in patients with diabetes.

\section{What's new}

- There is a higher rate of RAGE expression in diabetic patients compared to non-diabetics and patients with prostate cancer compared to BPH.

- Prognosis is worse in patients with prostate cancer with high RAGE expression.

\section{INTRODUCTION}

Type 2 Diabetes mellitus (DM) is chronic, a metabolic disease that has been associated with an increased risk of cancers including, pancreas, breast, lung, kidney, liver ${ }^{1-5}$. The association between diabetes and prostate cancer risk has been studied in several epidemiologic studies ${ }^{9-11}$. Some studies ${ }^{6-8}$ reported that prostate cancer had been inversely correlated with type 2 DM due to suppressed hypothalamic-pituitarytesticular axis. Otherwise, fewer population-based cohort studies indicated that history of diabetes mellitus was associated with an increased incidence of advanced prostate cancer related to high circulating levels of insulin, insulin resistance, or oxidative stress ${ }^{12-14}$.

Glycation is the non-enzymatic reaction between reducing sugars, such as glucose and proteins, lipids, or nucleic acids ${ }^{15}$. The formation of glycation products called AGE is a complicated process involving simple and more complex multistep reactions ${ }^{16}$. Endogenous formation of AGE can occur through three different pathways, including Maillard reactions, Polyol pathways, and lipid peroxidation ${ }^{17}$. Elevated levels of glucose accelerate the formation of AGEs. AGEs via their cognate receptor- the AGE specific receptor (RAGE)- can generate reactive oxygen species, activate inflammation signaling cascades and consequently play the role of development of diabetic complications ${ }^{18}$. AGE enhances several cancer cells by activating the cell cycle proteins (e.g., cyclin D1), anti-apoptotic proteins (e.g., BCL2) and they can contribute to development much cancer including gastric cancer, breast cancer, melanoma, pancreatic cancer and prostate cancer ${ }^{19-23}$.

To represent the potentially complex relationship between type $2 \mathrm{DM}$ and prostate cancer, we aimed to examine RAGE expression in localize and metastatic prostate cancer by using immunohistochemistry (IHC).

\section{MATERIALS AND METHODS}

\section{Study Population}

The prostate specimens of the study were obtained from the records of our university hospital. A total of 197 prostatic pre-operative biopsies and radical prostatectomy specimens from patients treated between 2009 and 2018 were studied. Of 197 samples, 87 belonged to localized prostate cancer, 46 to metastatic prostate cancer, and the remaining 64 specimens were from patients with benign prostate hyperplasia. All radical prostatectomy, TURP (Transurethral resection of the prostate) samples, and biopsies were initially evaluated by an expert pathologist and then radical prostatectomy, and biopsies specimens were graded according to the current international society of urological pathology (ISUP) grading system (Epstein et al. 2016). The Last update ISUP grade group has been considered the Gleason score: Grade Group 1= Gleason score [?]6, 
Grade Group 2= Gleason score 3+4=7, Grade Group 3= Gleason score 4+3=7, Grade Group 4= Gleason score 8, Grade Group $5=$ Gleason score [?]9, as well as modified morphological criteria for Gleason pattern $4^{24}$.

Detailed questionnaires were administered at baseline consisting of age, smoking, family history of prostate cancer, history of hypertension, and type 2 diabetes mellitus. Fasting height and weight were measured, and Body mass index (BMI) was calculated in all patients. Fasting Plasma Glucose (FPG mg/dL), HbA1C (\%, mmol/L), Total cholesterol (mg/dL), Low-Density Cholesterol (LDL, mg/dL), Triglycerides (mg/dL), Prostate-Specific Antigen (PSA) of all patients were recorded.

Imaging Techniques including Bone scan, Magnetic Resonance, and Computerized Tomography were performed in patients diagnosed with prostate cancer histopathologically after biopsy, according to D'amico risk classification and EAU (European Association of Urology) guidelines recommendations.

\section{Evaluation of recurrence in localized and metastatic prostate cancer}

For patients with localized prostate cancer, after radical prostatectomy, two consecutive serum PSA values higher than $0.2 \mathrm{ng} / \mathrm{mL}$ and/or increment were defined as biochemical recurrence (BCR). Currently, BCR has been accepted as the first rise $([?] 0.2 \mathrm{ng} / \mathrm{mL})$ in $\mathrm{PSA}^{25}$.

For patients with metastatic prostate cancer, castration resistance prostate cancer (CRPC) accepted for recurrence. CRPC is defined as castrating serum testosterone $<50 \mathrm{ng} / \mathrm{dl}$ or $1.7 \mathrm{nmol} / \mathrm{l}$ plus one of the following types of progression.

Biochemical progression: Three consecutive rises in PSA 1 week apart, resulting in two $50 \%$ increases over the nadir, and PSA $>2 \mathrm{ng} / \mathrm{ml}$.

Radiologic progression: The appearance of new lesions: either two or more new bone lesions on bone scan or a soft tissue lesion using the Response Evaluation Criteria in Solid Tumours (RECIST) ${ }^{26,27}$.

\section{Immunohistochemical assay}

Initially, pathological specimens were deparaffinized with dimethyl benzene and rehydrated through an ethanol gradient $(100 \%, 95 \%, 90 \%, 80 \%$, and $70 \%)$ into water. After washing with PBS (phosphate-buffered saline three times, slides were boiled in antigen retrieval buffer, $0.01 \mathrm{M}$ sodium citrate-hydrochloric acid $(\mathrm{pH}=6.0)$, for $30 \mathrm{~min}$ in a microwave oven. After endogenous peroxidase activity was quenched with $3 \%$ $\mathrm{H} 2 \mathrm{O} 2$ after three PBS washes, nonspecific antibody binding was blocked by incubating the slides with $10 \%$ normal goat nonimmune serum. The sections were then incubated at $4 \mathrm{degC}$ overnight with the rabbit polyclonal RAGE antibody (Santa-Cruz, CA, USA) at a 1:400 dilution and subsequently incubated with horseradish peroxidase (HRP) (ChemMateTM DAKO EnVisionTM Detection Kit) at room temperature for 30 min. After washing in PBS, the sections were then developed using 3,3-V-diaminobenzidine (Sigma), washed in running tap water, and lightly counterstained with hematoxylin before dehydration and coverslip mounting. Negative control experiments were conducted by replacing the primary antibody with PBS.

The RAGE detection was arbitrarily determined as to extent of immunoreactivity: $0,<5 \% ; 1,5 \%-10 \% ; 2$, $10 \%-50 \% ; 3,50 \%-75 \% ; 4,>75 \%$. Staining intensity was scored as 0 , negative; 1 , weak; 2 , moderate; 3 , strong (figure 1). For each case, the total immunohistostaining score, also known as the staining index (SI) was calculated by multiplying the percentage of positive cells with the staining intensity score, yielding a value between 0 and 12. For statistical analysis, staining intensity scores 0 and 1 defined as negative expression $(-), 2$ to 12 scores defined as positive expression $(+)^{28}$.

\section{Statistical Analyses}

Statistical analyses were performed using IBM SPSS Statistics for Windows, Version 23.0. a statistical software package (Armonk, NY: IBM Corp; 2015). Frequency distribution of demographic and clinicalpathological characteristics across the groups were compared using Pearson's chi-square tests. For comparison of continuous variables between two groups, the Student's t-test or Mann-Whitney U test was used, 
depending on whether or not the statistical hypotheses were fulfilled. Survival curves were generated using Kaplan-Meier methods, and the log-rank analysis was carried out to evaluate the survival differences between groups. All statistical tests were 2 -sided, and $\mathrm{p}<0.05$ was considered statistically significant.

\section{RESULTS}

Demographical and Biochemical and Immunohistochemical Parameters

Demographical, biochemical parameters and RAGE scores of three subgroups (BPH, LPCa, MetPCa) were demonstrated in table 1.

There were no significant RAGE expressions on the specimens of a total of 64 patients with BPH. RAGE expressions score of 24 diabetic and 40 non-diabetic patients with BPH were 0.5+-0.65 (range 0-4) and $0.6+-0.9$ (range 0-4), respectively (diabetic vs.non-diabetic $\mathrm{p}>0.05$ ). All biochemical parameters and RAGE scores of diabetic or non-diabetic patients with BPH were demonstrated in table 1.

There were 87 patients (diabetic 41; non-diabetic 46) with LPCa. RAGE scores of diabetic ( $\mathrm{n}=41)$ and nondiabetic patients $(\mathrm{n}=46)$ with LPCa were found to be $4.7+-3.1$ (range 1-12) and 1.5+-1.8 (range 0-9), respectively (diabetic vs. non-diabetic $\mathrm{p}=0.01$ ). All biochemical parameters and RAGE scores of diabetic or nondiabetic patients with LPCa were demonstrated in table 1. There were 46 (diabetic 30, non-diabetic 16) patients with MetPCa. RAGE scores of diabetic $(n=30)$ and nondiabetic patients $(n=16)$ with MetPCa were found to be 4.9+-3.6 (0-12) 1.6+-1.5 (range 0-6), (diabetic vs.non-diabetic, $\mathrm{p}=0.00$ ).

There was no difference between the patients' group (BPH, LPCa, MetPCa) for the age, fasting plasma glucose, HbA1c, and diabetes duration (except for diabetic patients).

\section{Correlations of RAGE expression with patients' survival rate}

There was a total of 46 patients with MetPca. Of 46, 27 patients had RAGE positive on specimens, and 19 had a negative expression on specimens. When compared each other with respect to cancer recurrence, cancer-specific mortality, and follow-up period (month); recurrence rate $(81,4 \%$ vs $78.9 \%, \mathrm{p}=0.8)$, cancerspecific mortality ( $33.3 \%$ vs $26.3 \%, \mathrm{p}=0.58$ ) were statistically found to be non -significant. (See table 2 , figure 2-3).

For the patients (total $n=87$ ) with localized prostate cancer, there were 47 patients with RAGE positive, 35 patients with RAGE negative. Five patients in the LPCa group had a positive surgical margin, so these patients were not included in the survival analysis. Comparison of these two groups patients, we did not find a significant difference for the cancer recurrence, duration of the follow-up, but we discovered that biochemical recurrence rate was found significantly higher in RAGE positive patients $(\mathrm{p}=0.00$; table 2 , figure 4$)$.

\section{DISCUSSION}

In the present study, we found an increased rate of RAGE expressions on the specimens of the localized and metastatic prostate cancer tissue samples to those of the patients with BPH. Additionally, we not only confirmed previous studies of RAGE expression in Prostate cancer samples but also compared the malignant prostate neoplasias (localized or metastatic) with benign prostate hyperplasia. A similar study has been previously reported on the overexpression of RAGE in patients with prostate neoplasias but not in a Turkish population $^{29}$.

The association between diabetes mellitus and the development of prostate cancer has been studied in numerous studies, but this relationship has been still controversial ${ }^{6,7,30,31}$. Some of these studies ${ }^{32,33}$ documented that there was a decreased risk of prostate cancer in patients with type 2 diabetes mellitus whereas; others documented an elevated risk of prostate cancer in these patients ${ }^{11,34,35}$. Lower androgen levels have been suggested as an explanation for the inverse correlation between diabetes and prostate cancer ${ }^{36}$. Furthermore, it was reported that low PSA levels were related to diabetes mellitus, and lower PSA values could cause fewer biopsies and result in decreased diagnose of prostate cancer in patients with type 2 diabetes mellitus ${ }^{37,38}$. Contrary to these studies ${ }^{35,38,45}$, in this study, we did not find low PSA levels in diabetic patients than 
non-diabetic patients $(\mathrm{p}=0.06)$. But we know that the measurement of PSA levels seems to be influenced by number factors including demographic, lifestyle, and health characteristics ${ }^{46}$. Variation of the serum PSA measurements could be an alternative explanation for the inverse correlation between diabetes and prostate cancer. On the other hand, preliminary studies were reporting that obesity, hyperinsulinemia, inflammatory cytokines, dyslipidemia, and common factors associated with type 2 DM were revealed as risk factors for various cancer types, including prostate cancer ${ }^{39}$.

It is well known that AGE and interactions of their receptor (RAGE) are produced increasingly in diabetes mellitus, and they induce oxidative stress, inflammatory reaction, apoptosis, autophagy which resultant with cancer development ${ }^{40-42}$. The previous study claimed that RAGE/NF- $\chi \mathrm{B}$ signaling pathways facilitated the prostate cancer metastasis ${ }^{43}$. They studied the HMGB1 (high mobility group box 1) and RAGE expression on 36 prostate tissue specimens (12 $\mathrm{BPH}, 12 \mathrm{LPCa}, 12 \mathrm{MetPCa}$ ) by immunohistochemically, and they showed the HMGB1 and RAGE expression were increased on tissues of LPCa and MetPCa and less detectable on muscles of BPH. In another study, they reported that RAGE expression was strongly correlated with progression and poor prognosis of prostate cancer ${ }^{44}$. They also found that RAGE's positive expression was significantly higher in prostate cancer than BPH $(\mathrm{p}=0.002)$. They suggested that the RAGE expressions were positively correlated with tumor stage, lymph node metastasis, and distant metastasis. Inconsistent with this study, the other study ${ }^{29}$ demonstrated that RAGE expression rates were significantly high on tissues of untreated prostate cancer and hormone-refractory prostate cancer tissue.

In the present study, we focused on diabetic patients because diabetes mellitus is the most known causative underlying factor in the occurrence of glycated end products and RAGE expression on tissues. Consistent with these regards, RAGE expressions were found to be more intense on prostate cancer tissues (localized or metastatic) among patients with Diabetes Mellitus than hyperplasia. When we compared the diabetic LPCa patients with diabetic MetPCa patients, RAGE expression on specimens of the metastatic disease was higher than localized cancer. But this was not significant statistically related to sample size. Our finding is consistent with the study ${ }^{44}$ mentioned above that overexpression of RAGE was strongly correlated with the metastatic diseases ${ }^{44,47}$. We could not find any significant difference in cancer recurrence, cancer metastasis, and clinical survival in diabetic and non-diabetic.

When we screen the studies related to the RAGE expression and cancer progress, the relationship between RAGE expression and patient outcome has still been a controversial issue. In certain cohort studies, it has been reported that there was a weak connection between the RAGE expression and cancer course of other cancer types, including colorectal, esophageal carcinoma ${ }^{48,49}$. Further investigations with a large number of patients and more extended follow-up periods may support our hypothesis with more satisfactory results.

One of the major limitations of this study is the small sample size for all three (BPH-LPCa-MetPCa) subgroups. Besides, this is a retrospective study, so some parameters, including a history of medications, smoking, are missing. Another limitation of our study is that diabetic and non-diabetic patients with prostate cancer (localized or metastatic) cannot be compared in terms of prognosis and survival because the number of diabetic patients is insufficient for statistical analysis.

In conclusion, In the present study, we found a high rate of RAGE expression in malignant prostate neoplasias (localized or metastatic) to the benign prostate hyperplasia, furthermore (beside) those with type 2 diabetes mellitus as expected revealed higher scores than the non-diabetics. Disease progression and survival parameters were worse in the patients with high RAGE levels, but it was not statistically significant. We already do not know the exact mechanisms and clinical consequences or any reasonable diabetic metabolic control on the prostate cancer prognosis. Further studies are needed.

\section{ACKNOWLEDGMENT}

The authors would like to thank the Cukurova University Research for funding this study.

\section{CONFLICTS OF INTEREST}

We declare that there was no conflict of interest. 


\section{AUTHOR CONTRIBUTIONS}

G. Akkus, V. Izol and F. Ok, data analysis/interpretation, critical revision of article, approval of article; M. Evran, M. Inceman and S. Erdogan, data analysis/interpretation, manuscript writing/editing; H. M. Kaplan, data extraction, statistics, data analysis/interpretation, manuscript writing/editing; M. Sert and T. Tetiker, concept/design, data analysis/interpretation, manuscript writing/editing.

\section{ETHICAL APPROVAL}

Permission was obtained from the Medical Research Ethics Committee of Cukurova University Faculty of Medicine for the study (approval number-March 4, 2016; 2/51).

This study was conducted in accordance with the Declaration of Helsinki in its latest version. This article does not contain any studies with animals performed by any of the authors.

\section{References}

1. Giovannuci E, Harlan DM, Archer MC, et al. Diabetes and cancer: a consensus report. Diabetes Care. 2010;33(7):1674-85.

2. Peairs KS, Barone BB, Snyder CF, et al. Diabetes mellitus and breast cancer outcomes: a systematic review and meta-analysis. J Clin Oncol. 2011; 29: 40-6.

3. Tan BX, Yao WX, Ge J, et al. Prognostic influence of metformin as first-line chemotherapy for advanced nonsmall cell lung cancer in patients with type 2 diabetes. Cancer 117(22): 5103-5111.

4. S.C. Larsson, A. Wolk, Diabetes mellitus and incidence of kidney cancer: a meta-analysis of cohort studies. Diabetologia 2011;54(5), 1013-1018.

5. Feng X, Wang G, Li N, et al. The association between fasting blood glucose and the risk of primary liver cancer in Chinese males: a population-based prospective study. Br J Cancer 2017; 117: 1405.

6. Fall K, Garmo H, Gudbjornsdottir S, et al. Diabetes mellitus and prostate cancer risk: a nationwide case-control study within PCBaSe Sweden. Cancer Epidemiol Biomarkers Prev 2013; 22: 1102-09.

7. Bonovas S, Filioussi K, Tsantes A. Diabetes mellitus and risk of prostate cancer: a meta-analysis. Diabetologia 2004; 47: 1071-78.

8. Kasper J, Giovannucci E. A meta-analysis of diabetes mellitus and the risk of prostate cancer. Cancer Epidemiol Biomarkers Prev 2006; 15: 2056

9. Lawrence YR, Morag O, Benderly M, et al. Association between metabolic syndrome, diabetes mellitus and prostate cancer risk. Prostate Cancer Prostatic Dis. 2013; 16: 181-186.

10. Rodriguez C, Patel AV, Mondul AM, Jacobs EJ, Thun MJ, Calle EE. Diabetes and risk of prostate cancer in a prospective cohort of US men. Am J Epidemiol. 2005; 161: 147-152.

11. Tseng CH. Diabetes and risk of prostate cancer: a study using the National Health Insurance. Diabetes Care. 2011; 34: 616-621.

12. Li Q, Kuriyama S, Kakizaki M, et al. History of diabetes mellitus and the risk of prostate cancer: the Ohsaki CohortStudy. Cancer Causes Control. 2010; 21: 1025-32

13. Peehl DM, Stamey TA. Serum-free growth of adult human prostatic epithelial cells. In Vitro Cell Dev Biol. 1986; 22(2):82-90.

14. Wright JL, Plymate SR, Porter MP, et al. Hyperglycemia and prostate cancer recurrence in men treated for localized prostate cancer. Prostate Cancer Prostatic Dis. 2013 Jun; 16(2):204-8.

15. Singh R, Barden A, Mori T, Beilin L. Advanced glycation end-products: a review. Diabetologia. 2001; 44(2):129-46.

16. Ahmed N, Thornalley PJ. Advanced glycation endproducts: what is their relevance to diabetic complications? Diabetes Obes Metab. 2007; 9(3):233-245

17. Monnier VM, Sell DR, Nagaraj RH, et al. Maillard reaction-mediated molecular damage to extracellular matrix and other tissue proteins in diabetes, aging, and uremia. Diabetes. 1992; 41 Suppl 2()$: 36-41$.

18. Goh SY, Cooper ME. Clinical review: The role of advanced glycation end products in progression and complications of diabetes. J Clin Endocrinol Metab. 2008; 93(4):1143-52. 
19. Li T, Qin W, Liu Y, et al. Effect of RAGE gene polymorphisms and circulating sRAGE levels on susceptibility to gastric cancer: a case-control study. Cancer Cell Int. 2017

20. Nankali M, Karimi J, Goodarzi M, et al. TIncreased Expression of the Receptor for Advanced Glycation End-Products (RAGE) Is Associated with Advanced Breast Cancer Stage. Oncol Res Treat. 2016; 39:622-628

21. Nguyen AH, Detty SQ, Agrawal DK. Clinical Implications of High-mobility Group Box-1 (HMGB1) and the Receptor for Advanced Glycation End-products (RAGE) in Cutaneous Malignancy: A Systematic Review. Anticancer Res. 2017; 37(1):1-7

22. Kang R, Tang D, Schapiro NE, et al. The HMGB1/RAGE inflammatory pathway promotes pancreatic tumor growth by regulating mitochondrial bioenergetics. Oncogene. 2014; 33:567-577.

23. Yang S, Pinney SM, Mallick P, et al. Impact of Oxidative Stress Biomarkers and Carboxymethyllysine (an AdvancedGlycation End Product) on Prostate Cancer: A Prospective Study. Clin Genitourin Cancer. 2015; 13(5):347-51

24. Epstein JI, Egevad L, Amin MB, et al. The 2014 International Society of Urological Pathology (ISUP) Consensus Conference on Gleason Grading of Prostatic Carcinoma: definition of grading patterns and proposal for a new grading system. Am J Surg Pathol (2016) 40(2):244-249

25. Moul JW: Prostate specific antigen only progression of prostate cancer. J Urol 2000; 163: 1632-1642.

26. Trock BJ, Han M, Freedland SJ, et al. Prostate cancer-specific survival following salvage radiotherapy vs observation in men with biochemical recurrence after radical prostatectomy. JAMA 2008; 299:27609.

27. Eisenhauer EA, Therasse P, Bogaerts J, et al. New response evaluation criteria in solid tumours: revised RECIST guideline (version 1.1). EJC 2009; 45:228-47.

28. Xu XC, Abuduhadeer X, Zhang WB, et al (2013). Knockdown of RAGE inhibits growth and invasion of gastric cancer Cells. Eur J Histochem, 29, 240-6.

29. Ishiguro H, Nakaigawa N, Miyoshi Y, Fujinami K, Kubota Y, Uemura H. Receptor for advanced glycation end products (RAGE) and its ligand, amphoterin are overexpressed and associated with prostate cancer development. The Prostate. 2005; 64:92-100.

30. Cai H, Xu Z, Xu T, et al. Diabetes mellitus is associated with elevated risk of mortality amongst patients with prostate cancer: a meta-analysis of 11 cohort studies. Diabetes Metab Res Rev. 2015; 31(4):336-43

31. Chen M, Luo Y, Yang S, et al. Association of diabetes mellitus with prostate cancer grade and prostatespecific antigen in Chinese biopsy population. Diabetes research and clinical practice 141 (2018): $80-87$.

32. Tsilidis KK, Allen NE, Appleby PN, et al. Diabetes mellitus and risk of prostate cancer in the European Prospective Investigation into Cancer and Nutrition. Int J Cancer 2015;136(2):372-81

33. Xu H, Jiang HW, Ding GX, et al. Diabetes mellitus and prostate cancer risk of different grade or stage: a systematic review and meta-analysis. Diabetes Clin Res Pract 2013; 99(3):241-9

34. Moreira DM, Anderson T, Gerber L, et al. The association of diabetes mellitus and high-grade prostate cancer in a multiethnic biopsy series. Cancer Causes Control. 2011; 22:977-983

35. Fukushima H, Masuda H, Kawakami S, et al. Effect of diabetes mellitus on high-grade prostate cancer detection among Japanese obese patients with prostate-specific antigen less than $10 \mathrm{ng} / \mathrm{mL}$. Urology. 2012; 79:1329-1334.

36. Betancourt-Albrecht M, Cunningham GR. Hypogonadism and diabetes. Int J Impot Res. 2003; 15(Suppl 4): S14-S20.

37. Peskoe SB, Joshu CE, Rohrmann S, et al. Circulating total testosterone and PSA concentrations in a nationally representative sample of men without a diagnosis of prostate cancer. Prostate. 2015; 75:1167-1176.

38. Barrett-Connor E. Lower endogenous androgen levels and dyslipidemia in men with non-insulindependent diabetes mellitus. Ann Intern Med. 1992; 117:807-811.

39. Mokdad AH, Ford ES, Bowman BA, et al. Prevalence of obesity, diabetes, and obesity-related health risk factors, 2001. Jama. 2003; 289:76-79.

40. Drinda S, Franke S, Ruster M, et al. Identification of the receptor for advanced glycation end products 
in synovial tissue of patients with rheumatoid arthritis. Rheumatol Int. 2005; 25:411-413.

41. Hu P, Lai D, Lu P, Gao J, He H. ERK and Akt signaling pathways are involved in advanced glycation end product-induced autophagy in rat vascular smooth muscle cells. Int J Mol Med. 2012; 29:613-618.

42. Yuan X, Zhang Z, Gong K, Zhao P, Qin J, Liu N. Inhibition of reactive oxygen species/extracellular signal-regulated kinases pathway by pioglitazone attenuates advanced glycation end products-induced proliferation of vascular smooth muscle cells in rats. Biol Pharm Bull. 2011; 34:618-623.

43. Zhang J, Shao S, Han D, et al. High mobility group box 1 promotes the epithelial-to-mesenchymal transition in prostate cancer PC3 cells via the RAGE/NF- $\chi \mathrm{B}$ signaling pathway. Int J Oncol. 2018; $53(2): 659-671$

44. Zhao C, Bao J, Lu Y, et al. Co-expression of RAGE and HMGB1 is associated with cancer progression and poor patient outcome of prostate cancer. Am J Cancer Res. American journal of cancer research 4.4 (2014): 369.

45. Baillargeon J, Pollock BH, Kristal AR, et al. The association of body mass index and prostate-specific antigen in a population-based study. Cancer. 2005; 103:1092-1095.

46. McGinley KF, McMahon GC, Brown GA. Impact of the US Preventive Services Task Force Grade D recommendation: assessment of evaluations for elevated prostate-specific antigen and prostate biopsies in a large urology group practice following statement revision. Rev Urol . 2015; 17(3):171-177.

47. Staquicini FI, Cardó-Vila M, Kolonin MG, et al. Vascular ligand-receptor mapping by direct combinatorial selection in cancer patients. Proc Natl Acad Sci U S A. 2011; 108(46):18637-42.

48. Tateno T, Ueno S, Hiwatashi K, et al. Expression of receptor for advanced glycation end products (RAGE) is related to prognosis in patients with esophageal squamous cell carcinoma. Ann Surg Oncol. 2009; 16(2):440-6

49. Fahmueller YN, Nagel D, Hoffmann RT, et al. Immunogenic cell death biomarkers HMGB1, RAGE, and DNAse indicate response to radioembolization therapy and prognosis in colorectal cancer patients. Int J Cancer 2013; 132(10):2349-58

TABLE 1 Demographic, biochemical, histopathological parameters and RAGE score of all three groups and subgroups (BPH-LPCa-MetPCa)

\begin{tabular}{|c|c|c|c|c|c|c|c|c|c|c|c|}
\hline & $\begin{array}{l}\mathrm{BPH} \\
(\mathrm{n}=64)\end{array}$ & $\begin{array}{l}\mathrm{BPH} \\
(\mathrm{n}=64)\end{array}$ & $\mathrm{p}^{*}$ & $\begin{array}{l}\mathrm{LPCa} \\
(\mathrm{n}=87)\end{array}$ & $\begin{array}{l}\mathrm{LPCa} \\
(\mathrm{n}=87)\end{array}$ & $\mathrm{p}^{* *}$ & $\begin{array}{l}\text { MetPCa } \\
(\mathrm{n}=46)\end{array}$ & $\begin{array}{l}\text { MetPCa } \\
(\mathrm{n}=46)\end{array}$ & $\mathrm{p}^{* * *}$ & $\begin{array}{l}\mathrm{P} \\
\text { Value }\end{array}$ & $\begin{array}{l}\mathrm{P} \\
\text { Value }\end{array}$ \\
\hline & $\begin{array}{l}\mathrm{DM} \\
(\mathrm{n}=24)\end{array}$ & $\begin{array}{l}\text { NDM } \\
(\mathrm{n}=40)\end{array}$ & & $\begin{array}{l}\mathrm{DM} \\
(\mathrm{n}=41)\end{array}$ & $\begin{array}{l}\text { NDM } \\
(\mathrm{n}=46)\end{array}$ & & $\begin{array}{l}\mathrm{DM} \\
(\mathrm{n}=30)\end{array}$ & $\begin{array}{l}\text { NDM } \\
(\mathrm{n}=16)\end{array}$ & & $\begin{array}{l}\mathrm{BPH} \\
\text { vs } \\
\mathrm{LPCa}\end{array}$ & $\begin{array}{l}\text { BPH vs } \\
\text { MetPCa }\end{array}$ \\
\hline $\begin{array}{l}\text { Age } \\
\text { (y) }\end{array}$ & $68.1 \pm 9.4$ & $67.0 \pm 8.6$ & $\mathrm{NG}$ & $60.2 \pm 3.4$ & $57.2 \pm 5.2$ & NG & $68.1 \pm 8.1$ & $70.5 \pm 7.6$ & NG & NG & NG \\
\hline $\begin{array}{l}\mathrm{BMI} \\
\left(\mathrm{kg} / \mathrm{m}^{2}\right)\end{array}$ & $28.2 \pm 3.3$ & $24.4 \pm 1.8$ & 0.001 & $28.2 \pm 2.6$ & $27.0 \pm 3.1$ & 0.06 & $26.9 \pm 3.5$ & $23.8 \pm 2.1$ & 0.001 & 0.001 & 0.94 \\
\hline $\begin{array}{l}\text { FPG } \\
(\mathrm{mg} / \mathrm{dL})\end{array}$ & $142.6 \pm 47$ & $.95 .4 \pm 10.1$ & 10.001 & $135.8 \pm 32$ & $.70 .4 \pm 7.0$ & 0.001 & $136.8 \pm 37$ & $.0 .9 \pm 9.4$ & 0.001 & 0.81 & 0.29 \\
\hline $\begin{array}{l}\mathrm{HbA1c} \\
(\%)\end{array}$ & $6.9 \pm 1.4$ & $5.3 \pm 0.3$ & 0.001 & $6.7 \pm 1.3$ & $5.4 \pm 0.3$ & 0.001 & $6.5 \pm 1.1$ & $5.3 \pm 0.3$ & 0.001 & 0.45 & 0.33 \\
\hline $\begin{array}{l}\text { DMt } \\
(y)\end{array}$ & & & & $7.7 \pm 4.1$ & & & $6.5 \pm 3.0$ & & & & \\
\hline $\begin{array}{l}\text { PSA } \\
(\mathrm{ng} / \mathrm{mL})\end{array}$ & $7.2 \pm 8.0$ & $5.2 \pm 7.7$ & 0.03 & $11.3 \pm 7.6$ & $11.9 \pm 12.4$ & 10.72 & $1075.0 \pm 2$ & $15 T 838 \pm 132$ & 28118 & 0.001 & 0.001 \\
\hline $\begin{array}{l}\text { RAGE } \\
\text { score }\end{array}$ & $0.5 \pm 0.6$ & $0.6 \pm 0.9$ & 0.69 & $4.7 \pm 3.1$ & $1.5 \pm 1.8$ & 0.01 & $4.9 \pm 3.6$ & $1.6 \pm 1.5$ & 0.001 & 0.001 & 0.001 \\
\hline
\end{tabular}

*: p value demonstrate the comparement of the diabetic and nondiabetic patients with BPH 
**: p value demonstrate the comparement of the diabetic and nondiabetic patients with LPCa

${ }^{* * *}: \mathrm{p}$ value demonstrate the comparement of the diabetic and nondiabetic patients with MetPCa

DM: Diabetes Mellitus, NDM: Non-Diabetes Mellitus NG: Non significant

BMI: body mass index, FPG: fasting plasma glucose, PSA: prostate specific antigen, DMt: Diabetes Mellitus Duration time

TABLE 2 Survival analysis of patients with metastatic and localized prostate cancer according to RAGE expression

\begin{tabular}{|c|c|c|c|}
\hline MetPCa & $\begin{array}{l}\text { RAGE Negative } \\
(\mathrm{n}=19)\end{array}$ & RAGE Positive $(\mathrm{n}=27)$ & p Value \\
\hline $\begin{array}{l}\text { Follow-up period } \\
\text { (months) } \pm \mathrm{SD}\end{array}$ & $49,6 \pm 36,7$ & $43,8 \pm 27,9$ & 0,54 \\
\hline Recurrence n (\%) & $15(78,9)$ & $22(81,4)$ & 0,83 \\
\hline $\begin{array}{l}\text { Mean RFS (months) } \pm \\
\text { SE (\%95 CI) }\end{array}$ & $32.7 \pm 7.6(17.8-47.7)$ & $25.9 \pm 4.7(16.6-35.2)$ & 0,55 \\
\hline 1 -year RFS $(\%) \pm \mathrm{SE}$ & $63,2 \pm 11,1$ & $67,6 \pm 9,5$ & \\
\hline 3 -year RFS $(\%) \pm \mathrm{SE}$ & $35,5 \pm 11,2$ & $10,6 \pm 6,9$ & \\
\hline $\begin{array}{l}\text { All-cause mortality n } \\
(\%)\end{array}$ & $6(31,6)$ & $9(33,3)$ & 0,90 \\
\hline $\begin{array}{l}\text { Mean OS (months) } \pm \\
\text { SE }(\% 95 \mathrm{CI})\end{array}$ & $79,2 \pm 10,1 \quad(59,4-98,9)$ & $74,9 \pm 7,9(59,3-90,5)$ & 0,65 \\
\hline 3 -year OS $(\%) \pm \mathrm{SE}$ & $68,7 \pm 11,8$ & $80,2 \pm 9,1$ & \\
\hline 5 -year OS $(\%) \pm \mathrm{SE}$ & $68,7 \pm 11,8$ & $71,3 \pm 11,6$ & \\
\hline $\begin{array}{l}\text { Cancer-specific } \\
\text { mortality n }(\%)\end{array}$ & $5(26,3)$ & $9(33,3)$ & 0,58 \\
\hline $\begin{array}{l}\text { Mean CSS (months) } \pm \\
\text { SE (\%95 CI) }\end{array}$ & $83,4 \pm 9,8(64,2-10,7)$ & $74,9 \pm 7,9(59,3-90,5)$ & 0,43 \\
\hline 3 -year CSS $(\%) \pm \mathrm{SE}$ & $73,2 \pm 11,7$ & $80,2 \pm 9,1$ & \\
\hline $\begin{array}{l}5 \text {-year CSS }(\%) \pm \mathrm{SE} \\
\mathbf{L P C a}\end{array}$ & $\begin{array}{l}73,2 \pm 11,7 \\
(\mathrm{n}=35)\end{array}$ & $\begin{array}{l}71,3 \pm 11,6 \\
(\mathrm{n}=47)\end{array}$ & \\
\hline $\begin{array}{l}\text { Follow-up period } \\
\text { (months) } \pm \mathrm{SD}\end{array}$ & $54,5 \pm 12,1$ & $57,5 \pm 12,8$ & 0,30 \\
\hline BCR n $(\%)$ & $3(8,6)$ & $8(17)$ & 0,00 \\
\hline $\begin{array}{l}\text { Mean BCRFS } \\
\text { (months) } \pm \text { SE }(\% 95 \\
\text { CI) }\end{array}$ & $70.4 \pm 1.9(66.6-74.2)$ & $63,1 \pm 3,2(56,9-69,3)$ & 0,26 \\
\hline $\begin{array}{l}\text { 3-year BCRFS }(\%) \pm \\
\text { SE }\end{array}$ & $97,1 \pm 2,8$ & $87,2 \pm 4,9$ & \\
\hline $\begin{array}{l}5 \text {-year BCRFS }(\%) \pm \\
\text { SE }\end{array}$ & $85,6 \pm 8,9$ & $80,5 \pm 6,5$ & \\
\hline
\end{tabular}

SD; standard deviation, SE; standard error, RFS; recurrence free survival, OS; overall survival, CSS; cancer specific survival BCR; biochemical recurrence, BCRFS; biochemical recurrence free survival, CI; confidence interval

Figure legends

FIGURE 1 Examples of Immunohistochemical staining (IHC) of RAGE in BPH and adenocarcinoma tissues. 
Negative expression of RAGE in BPH (RAGE scoring=0)10xmagnification

1a) Negative expression of RAGE in adenocarcinoma (RAGE scoring=0) 20xmagnification 1b) Weak positive expression of RAGE in adenocarcinoma (RAGE scoring=1) 20xmagnification 1c)Moderate positive expression of RAGE in adenocarcinoma (RAGE scoring=2) 10xmagnification 1d)Strong positive expression of RAGE in adenocarcinoma (RAGE scoring $=3$ ) 20xmagnification

FIGURE 2 Kaplan-Meier survival curves for cancer specific survival analysis of RAGE groups in metastatic prostate cancer. Log-rank test $\mathrm{p}=0,43$.

FIGURE 3 Kaplan-Meier survival curves for recurrence-free survival analysis of RAGE groups in metastatic prostate cancer. Log-rank test $\mathrm{p}=0,55$.

FIGURE 4 Kaplan-Meier survival curves for biochemical recurrence-free survival analysis of RAGE groups in localized prostate cancer. Log-rank test $\mathrm{p}=0,26$. 


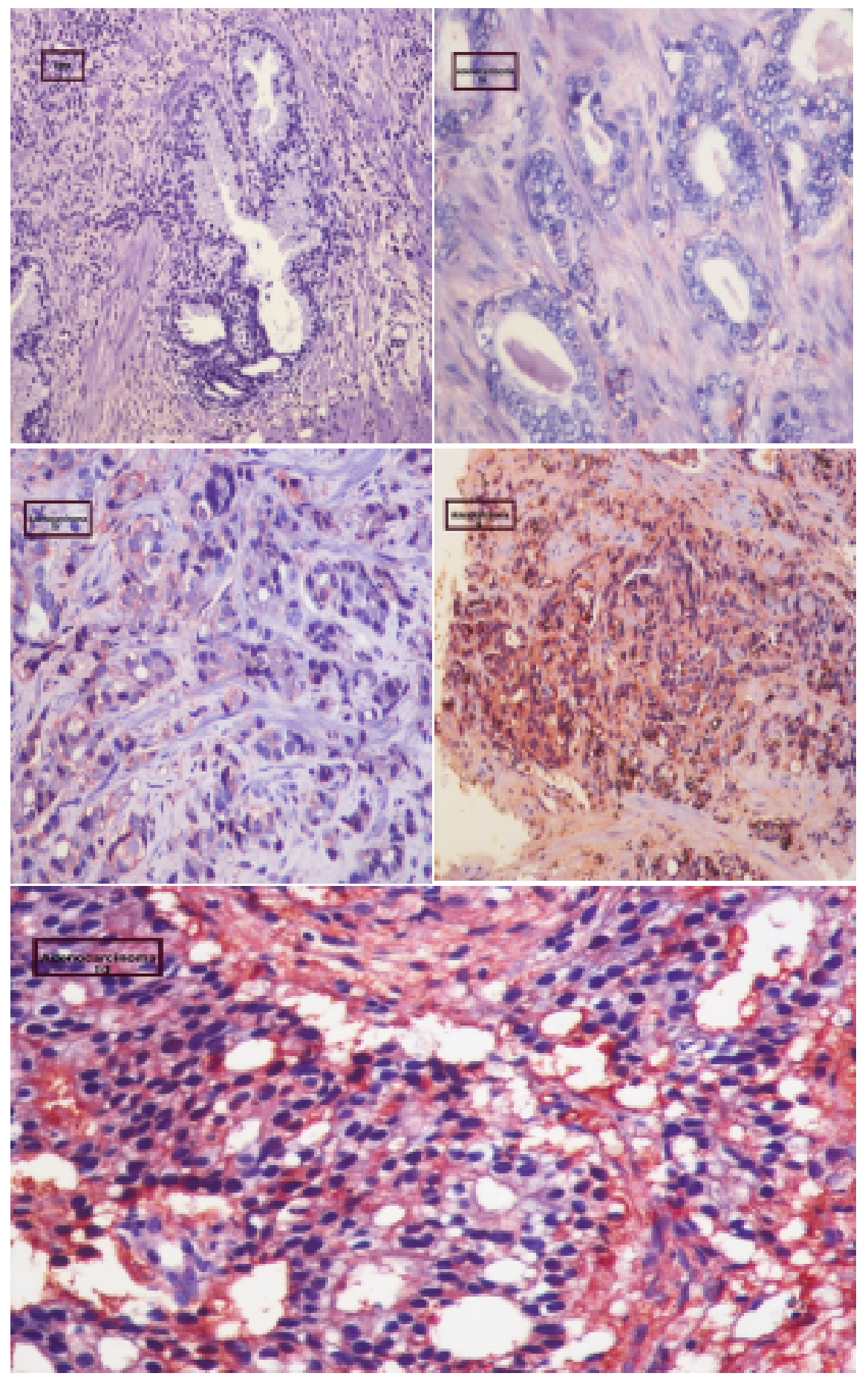



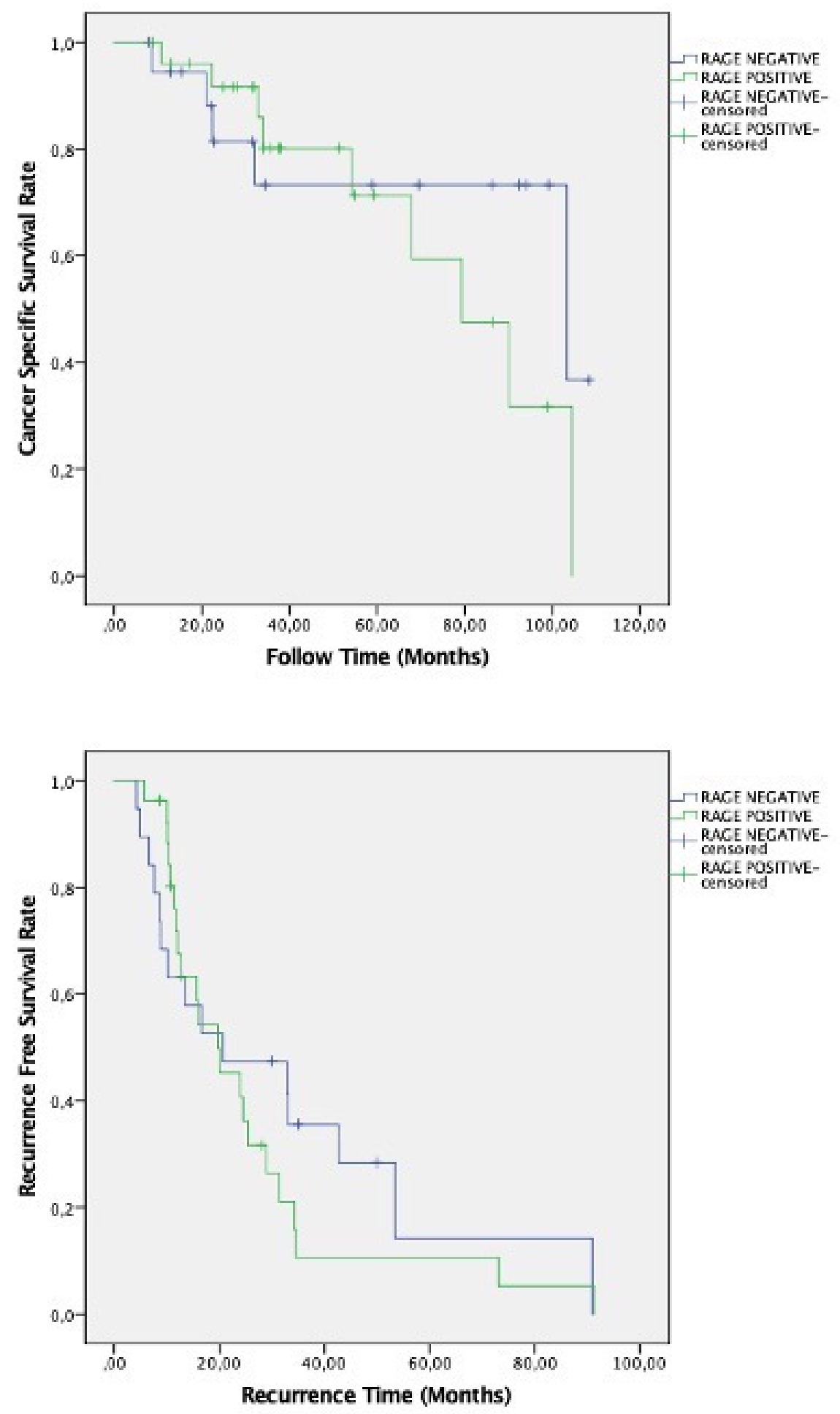


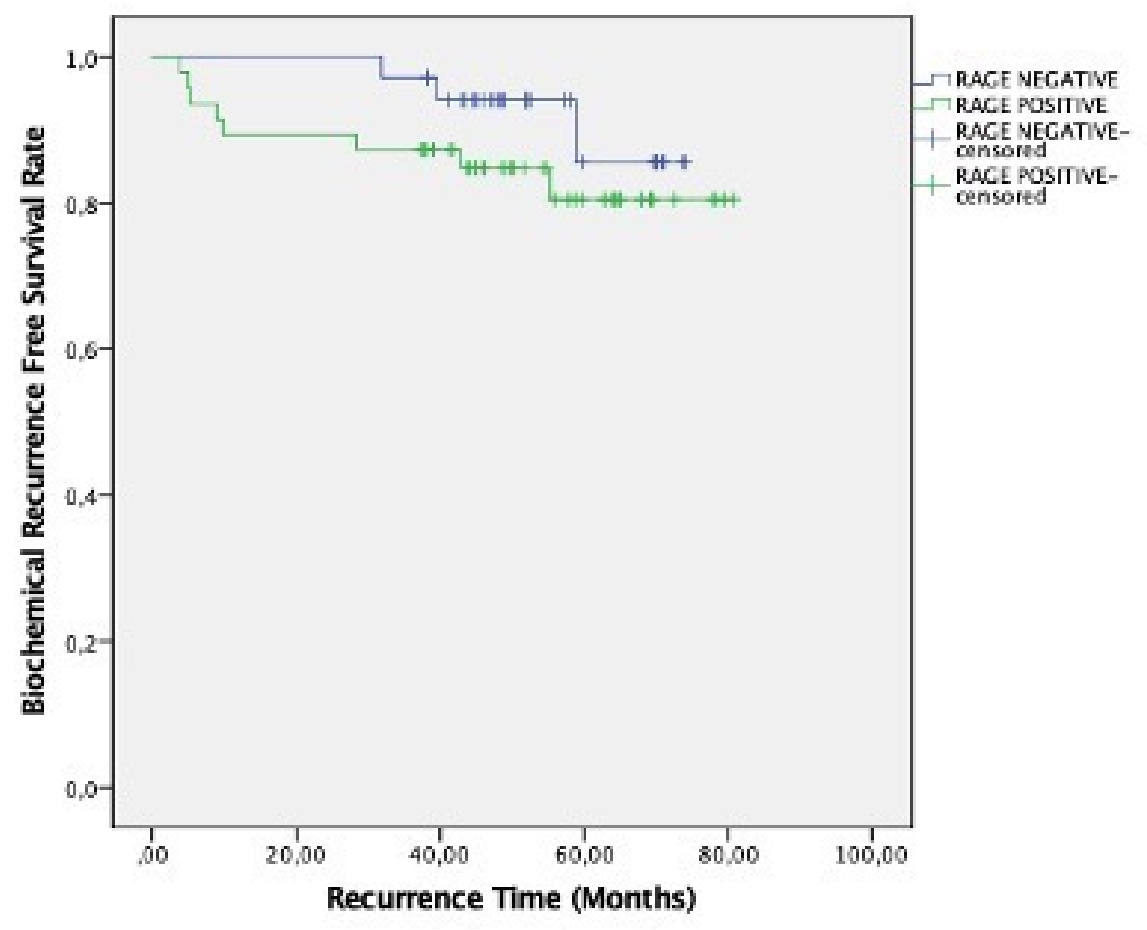

